\title{
Prevalence, risk factors and impact on outcomes of 30-day unexpected rehospitalization in incident peritoneal dialysis patients
}

Jianbo $\mathrm{Li}^{1,2+}$, Jing $\mathrm{Yu}^{1,2+}$, Naya Huang ${ }^{1,2}$, Hongjian Ye ${ }^{1,2}$, Dan Wang ${ }^{1,2}$, Yuan Peng ${ }^{1,2}$, Xiaobo Guo ${ }^{3}$, Chunyan $\mathrm{Yi}^{1,2}$, Xiao Yang ${ }^{1,2}$ and Xueqing $Y u^{1,2,4,5^{*}}$ (i)

\begin{abstract}
Background: Rehospitalization is a major problem for end stage renal disease (ESRD) populations. However, researches on 30-day unexpected rehospitalzation of incident peritoneal dialysis (PD) patients were limited. This study aimed to investigate the prevalence, risk factors and impact on outcomes of 30-day unexpected rehospitalization in incident PD patients.

Methods: This was a retrospective cohort study. Patients who accepted PD catheter implantation in our centre from Jan 1, 2006 to Dec 31, 2013 and regular follow-up were included. The demographic characteristics, laboratory parameters, and rehospitalization data were collected and analyzed. The primary outcome was all-cause mortality, and the secondary outcomes included cardiovascular disease (CVD) mortality and technical failure.

Results: Totally 1632 patients ( $46.9 \pm 15.3$ years old, 60.1\% male, 25.6\% with diabetes) were included. Among them, 149 (9.1\%) had a 30-day unexpected rehospitalization after discharge. PD-related peritonitis $(n=48,32.2 \%)$, catheter malfunction ( $n=30,20.1 \%)$ and severe fluid overload $(n=19,12.8 \%)$ were the top three causes for the rehospitalization. Multivariate logistic regression analysis showed that length of index hospital stays [Odds ratio (OR) $=1.02,95 \%$ confidence interval $(C \mathrm{Cl}) 1.00-1.03, P=0.036)$ and hyponatremia $(\mathrm{OR}=1.85,95 \% \mathrm{Cl} 1.06-3.24, P=0.031)$ were independently associated with the rehospitalization. Multivariate Cox regression analysis indicated that 30-day rehospitalization was an independent risk factor for all-cause mortality [Hazard ratio (HR) $=1.52,95 \% \mathrm{Cl} 1.07-2.16$, $P=0.019)$ and $C V D$ mortality $(H R=1.73,95 \% \mathrm{Cl} 1.03-2.90, P=0.038)$.

Conclusions: The prevalence of 30-day unexpected rehospitalization for incident PD patients in our centre was 9.1\%. The top three causes for the rehospitalization were PD-related peritonitis, catheter malfunction and severe fluid overload. Thirty-day unexpected rehospitalization increased the risk of all-cause mortality and CVD mortality for PD patients.
\end{abstract}

Keywords: Prevalence, Risk factors, Outcomes, 30-day unexpected rehospitalization, Peritoneal dialysis

\footnotetext{
* Correspondence: yuxq@mail.sysu.edu.cn

${ }^{\dagger}$ Jianbo Li and Jing Yu contributed equally to this work.

'Department of Nephrology, The First Affiliated Hospital, Sun Yat-sen University, Guangzhou 510080, Guangdong, China

${ }^{2}$ Key Laboratory of Nephrology, National Health Commission and Guangdong Province, Guangzhou 510080, Guangdong, China

Full list of author information is available at the end of the article
}

(c) The Author(s). 2020 Open Access This article is licensed under a Creative Commons Attribution 4.0 International License, which permits use, sharing, adaptation, distribution and reproduction in any medium or format, as long as you give appropriate credit to the original author(s) and the source, provide a link to the Creative Commons licence, and indicate if changes were made. The images or other third party material in this article are included in the article's Creative Commons licence, unless indicated otherwise in a credit line to the material. If material is not included in the article's Creative Commons licence and your intended use is not permitted by statutory regulation or exceeds the permitted use, you will need to obtain permission directly from the copyright holder. To view a copy of this licence, visit http://creativecommons.org/licenses/by/4.0/ The Creative Commons Public Domain Dedication waiver (http://creativecommons.org/publicdomain/zero/1.0/) applies to the data made available in this article, unless otherwise stated in a credit line to the data. 


\section{Background}

Rehospitalization is a major problem for end stage renal disease (ESRD) populations [1-3]. According to the 2018 United States Renal Data System (USRDS) report, about $35.4 \%$ of ESRD patients have an unplanned rehospitalization within 30 days after discharge [1]. Also, rehospitalizations are associated with increased morbidity and mortality and reduced quality of life among dialysis patients [1-3]. Furthermore, inpatient treatment poses a significant financial burden for Medicare expenditures and patients. In 2016, nearly 12 billion dollars had been paid for inpatient care of ESRD patients, accounting for approximately $33 \%$ of the total Medicare expenditures for them [1].

Peritoneal dialysis (PD) is a commonly used method of renal replacement therapy for ESRD patients. The readmission rates of PD patients were quite high, $15.5-37.4 \%$, as reported in developed countries $[1,4$, 5]. Although several studies have investigated the risk factors and prevention strategies of readmission among hemodialysis (HD) patients [6-9], evidence regarding the prevalence and modifiable risk factors of 30-day readmission among PD patients in developing countries was still limited. In addition, the association of rehospitalization and long-term outcomes among these patients had rarely been described.

In this study, we aimed to investigate the prevalence, causes and risk factors of 30-day unexpected rehospitalization among incident PD patients as well as the association between the rehospitalization and long-term outcomes.

\section{Methods}

\section{Study design and population}

This was a retrospective, single-centre cohort study. Patients who received PD catheter implantation in the Department of Nephrology, the First Affiliated Hospital of Sun Yat-sen University from Jan 1, 2006 to Dec 31, 2013, age $\geq 18$ years, and regular follow-up were included. Patients who dropped out during index hospitalization, presenting with a history of malignancy or kidney transplantation, transfer from hemodialysis, or with incomplete data were excluded. Catheter implantation was done with open laparotomy technique by experienced nephrologists following the same procedure $[10,11]$. And the implantation was performed by different nephrologists during the eightyear study. All patients were followed until death, withdrawal from PD, or until Aug 31, 2016. The study protocol was approved by the Ethics Committee of The First Affiliated Hospital of Sun Yat-sen University. All participants signed written informed consent forms.

\section{Demographic and clinical data}

Demographic, clinical and laboratory data were collected. Baseline demographic data included age, gender, primary kidney diseases, diabetes mellitus (DM), cardiovascular diseases (CVD) history. CVD was defined as arrhythmias, valvular heart disease, congestive heart failure, angina, myocardial infarction, transient ischemic attack, stroke, or peripheral arterial disease [12]. Clinical data included body mass index (BMI), mean blood pressure, and length of index hospital stay. Baseline laboratory data closest to the discharge date were collected, which included hemoglobin, platelet count, uric acid, corrected calcium, phosphorus, intact parathyroid hormone (iPTH), albumin, total cholesterol (TC), triglyceride (TG), hyponatremia, hypokalemia and creatinine. Corrected calcium was calculated by the conventional Payne equation (c [Ca $] \mathrm{mmol} / \mathrm{L}=\mathrm{t} \quad[\mathrm{Ca}]+0.02 \times[40-$ albumin $\mathrm{g} / \mathrm{L}])$ [13]. Hyponatremia was defined as serum sodium level $<135 \mathrm{mmol} / \mathrm{L}$ [14]. Hypokalemia was defined as serum potassium level $<3.5 \mathrm{mmol} / \mathrm{L}$ [15]. The serum sodium and potassium were measured in the clinical laboratory of our hospital using the indirect ion electrode method (AU5800, Beckman Coulter Inc., Brea, CA, USA).

The main causes for 30-day unexpected rehospitalization were also collected from patients' files, which were classified as PD-related peritonitis [16], catheter malfunction [11], severe fluid overload (excluding congestive heart failure as it was assigned to CVD) [17], non-peritonitis infection, CVD events [12], abdominal wall hernia [18], refractory hypertension [19] and other causes. The hospitalization in which patients obtained PD catheterization and began PD treatment was defined as the index hospitalization. Thirty-day unexpected rehospitalization was defined as rehospitalization for unexpected clinical events within 30 days after discharge [1]. We excluded emergency visits and scheduled rehospitalizations for routine examination, intravenous iron supplementation, cyclophosphamide pulse therapy, or other planned procedures.

\section{Outcomes}

The primary outcome of this study was all-cause mortality, and the secondary outcomes included CVD mortality and death-censored technical failure. CVD mortality was defined as mortality caused by CVD events [12]. Deathcensored technical failure was defined as transfer to HD for more than 90 days from any cause, and it was censored for death, spontaneous recovery of renal function, move to another centre, kidney transplantation, and/or “still on PD" [20] until Aug 31, 2016. 


\section{Statistical analysis}

Quantitative variables were displayed as the mean \pm standard deviation (SD) for normal distribution and the median (interquartile range, IQR) for skewed distribution. Qualitative variables were expressed as frequencies and percentages. Normally distributed variables were compared using the t-test, and asymmetrically distributed variables were compared using the Wilcoxon rank sum test. Comparisons of categorical variables were tested by the chi-square test. The multivariate logistic regression model was used to identify the independent risk factors that were associated with 30-day unexpected rehospitalization. Survival curves were generated by the Kaplan-Meier method. Multivariate Cox regression models were used to evaluate the association between 30-day unexpected rehospitalization and all-cause mortality, CVD mortality and technical failure. $P<0.05$ was considered statistical significance. Statistical analyses were performed using SPSS version 19.0 (SPSS Inc., Chicago, IL, USA).

\section{Results}

\section{Characteristics of the study cohort}

A total of 1885 incident PD patients were screened, and finally 1632 patients were included in this study (Fig. 1). The demographic, clinical and laboratory characteristics of the study cohort are shown in Table 1 . The average age was $46.9 \pm 15.3$ years old, males accounted for $60.1 \%$ $(n=981)$, and $25.6 \%(n=417)$ had DM. Among these patients, 9.1\% $(n=149)$ had an unexpected rehospitalization within 30 days after discharge from the index hospitalization, including 2.9\% (48/1632) PD-related peritonitis, $1.8 \%(30 / 1632)$ catheter malfunction, $1.2 \%$ (19/1632) severe fluid overload, $1.0 \%$ (17/1632) nonperitonitis infection, $0.7 \%$ CVD events (12/1632), 0.2\% (4/1632) abdominal wall hernia, $0.1 \%(2 / 1632)$ refractory hypertension and $1.0 \%(17 / 1632)$ other causes. PDrelated peritonitis $(48 / 149,32.2 \%)$, catheter malfunction $(30 / 149,20.1 \%)$ and severe fluid overload (19/149, $12.8 \%)$ were the top three causes for rehospitalization (Fig. 2). Among the 48 peritonitis-related rehospitalizations, $22(45.8 \%)$ were culture negative, $2(4.2 \%)$ had no data, $8(16.7 \%)$ were caused by Escherichia coli, 4(8.3\%) were Staphylococcus aureus, 3 were Streptococcus, another 3 were Staphylococcus epidermidis, 2 were Klebsiella pneumoniae, the rest 4 were other bacteria. And among these peritonitis episodes, $11(22.9 \%)$ were related to patient's techniques, which defined as unqualified operating environment or unqualified operating methods. Fifteen episodes of catheter malfunction were caused by omental wrap which resolved by omental release surgery, 11 were caused by functional catheter shift which resolved by conservative treatment, 2 were caused by catheter shift which resolved by catheter reposition surgery, and 2 were caused by other reasons.

Compared with those who did not have 30-day unexpected rehospitalization (group 1), patients in the rehospitalization group (group 2) were older [50.2 \pm 16.8 years

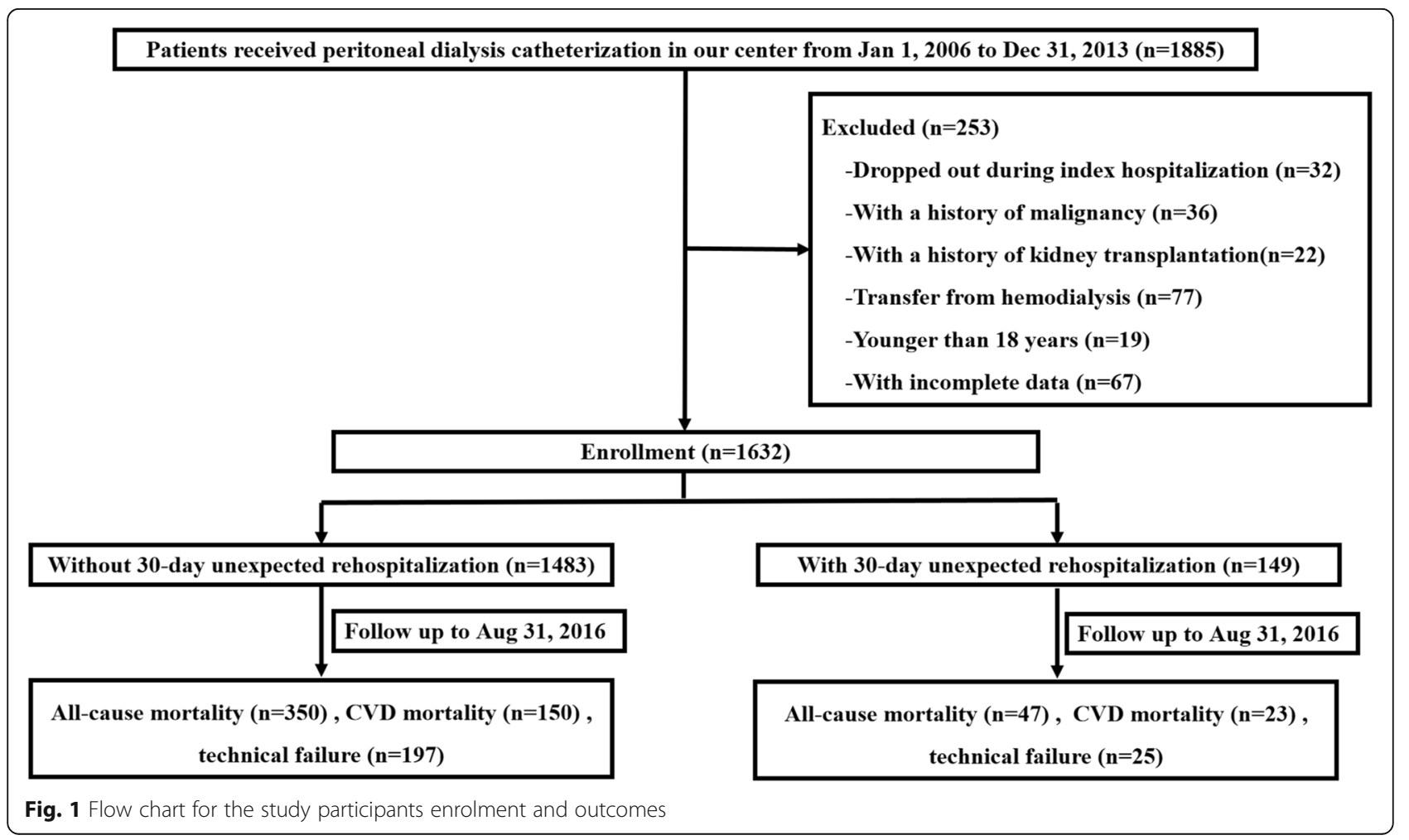


Table 1 Comparison of baseline parameters between patients without (group 1) and with (group 2) 30-day unexpected rehospitalization

\begin{tabular}{|c|c|c|c|c|}
\hline Variables & Total $(n=1632)$ & Group $1(n=1483)$ & Group $2(n=149)$ & $P$ value \\
\hline Age (years) & $46.9 \pm 15.3$ & $46.6 \pm 15.2$ & $50.2 \pm 16.8$ & 0.013 \\
\hline Gender (male) n (\%) & $981(60.1)$ & $892(60.1)$ & $89(59.7)$ & 0.921 \\
\hline Primary kidney diseases n (\%) & & & & 0.331 \\
\hline Chronic glomerulonephritis & $991(60.7)$ & $905(61.1)$ & $85(57.0)$ & - \\
\hline Diabetic nephropathy & $365(22.4)$ & $324(21.8)$ & $41(27.5)$ & - \\
\hline Hypertensive nephropathy & $113(6.9)$ & $106(7.1)$ & $7(4.7)$ & - \\
\hline Others & $163(10.0)$ & $147(9.9)$ & $16(10.7)$ & - \\
\hline Diabetes mellitus n (\%) & $417(25.6)$ & $371(25.0)$ & $46(30.9)$ & 0.118 \\
\hline CVD history n (\%) & $348(21.3)$ & $313(21.1)$ & $35(23.5)$ & 0.498 \\
\hline $\mathrm{BMI}\left(\mathrm{kg} / \mathrm{m}^{2}\right)$ & $21.8 \pm 3.2$ & $21.8 \pm 3.2$ & $22.0 \pm 3.2$ & 0.372 \\
\hline Mean blood pressure (mmHg) & $110.4 \pm 16.8$ & $109.0 \pm 16.7$ & $110.0 \pm 18.5$ & 0.760 \\
\hline Length of index hospital stay (day) $)^{a}$ & $23.7 \pm 10.1$ & $23.5 \pm 9.9$ & $26.3 \pm 12.0$ & 0.006 \\
\hline Hemoglobin $(g / L)$ & $79.2 \pm 18.7$ & $79.2 \pm 18.7$ & $78.3 \pm 18.1$ & 0.539 \\
\hline Platelet $\left(10^{9} / \mathrm{L}\right)$ & $193.6 \pm 78.7$ & $193.9 \pm 79.2$ & $190.6 \pm 73.4$ & 0.627 \\
\hline Uric acid ( $\mu \mathrm{mol} / \mathrm{L})$ & $488.5 \pm 149.5$ & $490.2 \pm 148.7$ & $471.7 \pm 157.0$ & 0.161 \\
\hline Corrected calcium (mmol/L) & $2.1 \pm 0.3$ & $2.1 \pm 0.3$ & $2.1 \pm 0.3$ & 0.290 \\
\hline Phosphorus (mmol/L) & $2.0 \pm 0.6$ & $2.1 \pm 0.6$ & $2.0 \pm 0.5$ & 0.196 \\
\hline iPTH (pg/mL) & $\begin{array}{l}348.7 \\
(201.7540 .2)\end{array}$ & $\begin{array}{l}351.8 \\
(204.7539 .0)\end{array}$ & $\begin{array}{l}333.9 \\
(175.2551 .2)\end{array}$ & 0.521 \\
\hline Albumin (g/L) & $34.7 \pm 5.1$ & $34.8 \pm 5.1$ & $33.8 \pm 5.7$ & 0.016 \\
\hline TC $(\mathrm{mmol} / \mathrm{L})$ & $4.7 \pm 1.4$ & $4.7 \pm 1.4$ & $4.7 \pm 1.6$ & 0.802 \\
\hline $\mathrm{TG}(\mathrm{mmol} / \mathrm{L})$ & $1.3(0.9,1.9)$ & $1.3(0.9,1.9)$ & $1.3(1.0,2.0)$ & 0.664 \\
\hline Hyponatremia n (\%) & $105(6.9)$ & $87(6.3)$ & $18(12.9)$ & 0.003 \\
\hline Hypokalemia n (\%) & $364(23.8)$ & $328(23.6)$ & $36(25.7)$ & 0.578 \\
\hline Creatinine $(\mu \mathrm{mol} / \mathrm{L})$ & $793.7 \pm 286.9$ & $794.8 \pm 283.6$ & $782.1 \pm 318.9$ & 0.648 \\
\hline
\end{tabular}

Values were expressed as mean $\pm \mathrm{SD}$, median (interquartile range), or number (percentage)

Abbreviations: CVD cardiovascular disease, BMI body mass index, IPTH intact parathyroid hormone, TC total cholesterol, TG triglyceride

${ }^{a}$ Length of index hospital stay refers to the hospitalization days during which patients obtained peritoneal dialysis (PD) catheterization and began PD treatment

verse (vs.) $46.6 \pm 15.2$ years, $P=0.013)]$, had longer length of index hospital stay ( $26.3 \pm 12.0$ days vs. $23.5 \pm$ 9.9 days, $P=0.006)$, lower level of albumin $(33.8 \pm 5.7 \mathrm{~g} / \mathrm{L}$ vs. $34.8 \pm 5.1 \mathrm{~g} / \mathrm{L}, P=0.016$ ), higher proportion of hyponatremia $[12.9 \%$ vs. $6.3 \%, P=0.003]$. However, there were no significant differences in gender, causes of primary kidney diseases, DM, CVD history, BMI, mean blood pressure level, and levels of hemoglobin, platelet, uric acid, corrected calcium, phosphorus, iPTH, TC, TG, creatinine, and proportion of hypokalemia between the two groups.

\section{Risk factors associated with unexpected 30-day rehospitalization}

Univariate logistic regression analysis revealed that advanced age, longer length of index hospital stay and hyponatremia were positively correlated with the rehospitalization, and albumin level was negatively correlated with the rehospitalization (Table 2). Multivariate logistic regression analysis showed that length of index hospital stay [Odds ratio $(\mathrm{OR})=1.02,95 \%$ confidence interval (CI) 1.00-1.03, $P=0.036$ ] and hyponatremia $(\mathrm{OR}=1.85,95 \%$ CI $1.06-3.24, P=0.031)$ were positively independently associated with 30-day unexpected rehospitalization after adjusting for age, gender, diabetes mellitus, CVD history and albumin (Table 2).

\section{Association of unexpected 30-day rehospitalization with clinical outcomes}

After a median of $34.5(15.0,55.0)$ months' follow-up, $397(24.3 \%)$ patients died, of which $173(10.6 \%)$ were caused by CVD, and 222 (13.6\%) patients had technical failure. Patients in the rehospitalization group had higher rates of all-cause mortality $(31.5 \%$ vs. $23.6 \%, P=0.031)$ and CVD mortality ( $15.4 \%$ vs. $10.1 \%, P=0.044)$ compared with the non-rehospitalization group. The proportion of technical failure between the two groups was comparable $(16.8 \%$ vs. $13.3 \%, P=0.236)$. Survival curves 


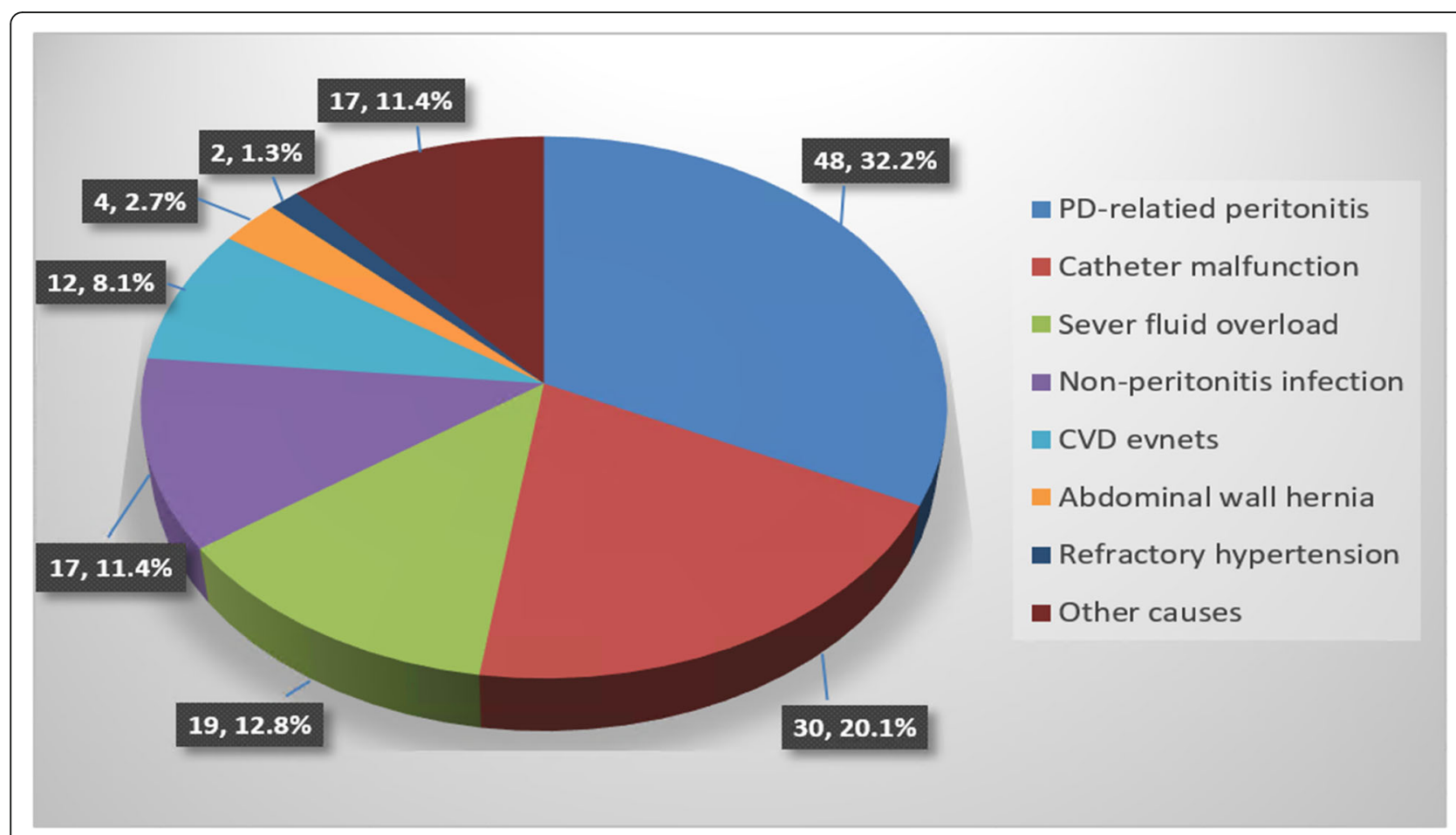

Fig. 2 Main causes of 30-day unexpected rehospitalization

showed that the cumulative incidence of all-cause mortality (Log Rank $X^{2}=15.731, P<0.001$ ), CVD mortality (Log Rank $\left.\chi^{2}=11.873, P=0.001\right)$ and technical failure (Log Rank $\left.X^{2}=7.245, P=0.007\right)$ were significantly higher in the rehospitalization group compared with the nonrehospitalization group (Fig. 3). Multivariate Cox regression analysis showed that 30-day unexpected rehospitalization was an independent risk factor for all-cause mortality [Hazard ratio (HR) $=1.52$, 95\% CI 1.07-2.16, $P=0.019)$ after adjusting for age, gender, diabetes mellitus, CVD history, BMI, length of index hospital stay, hemoglobin, platelet, corrected calcium, phosphorus, $\mathrm{iPTH}$, albumin, TC, TG, hyponatremia, hypokalemia, uric acid and creatinine, and for CVD mortality $(\mathrm{HR}=$ 1.73, 95\% CI 1.03-2.90, $P=0.038$ ) after adjusting for age, gender, diabetes mellitus, CVD history, BMI, length of index hospital stay, hemoglobin, platelet, corrected calcium, phosphorus, iPTH, albumin, TC, TG, uric acid and creatinine (Table 3).

\section{Discussion}

In the present study, we found that the prevalence of 30-day unexpected rehospitalization in incident PD patients was $9.1 \%$ in our PD centre. The top three causes for the rehospitalization were PD-related peritonitis, catheter malfunction and severe fluid overload. Length

Table 2 Factors associated with 30-day unexpected rehospitalization in the logistic regression model

\begin{tabular}{|c|c|c|c|c|}
\hline \multirow[t]{2}{*}{ Variables } & \multicolumn{2}{|l|}{ Univariate } & \multicolumn{2}{|l|}{ Multivariate } \\
\hline & $\mathrm{OR}(95 \% \mathrm{Cl})$ & $P$ value & OR(95\%Cl) & $P$ value \\
\hline$\overline{\text { Age (every } 1 \text { year } \uparrow)}$ & $1.02(1.00,1.03)$ & 0.007 & $1.01(1.00,1.03)$ & 0.091 \\
\hline Gender (female vs. male) & $1.02(0.72,1.44)$ & 0.921 & $1.08(0.75,1.54)$ & 0.691 \\
\hline Diabetes mellitus (yes vs.no) & $1.34(0.93,1.93)$ & 0.119 & $1.08(0.70,1.67)$ & 0.738 \\
\hline CVD history (yes vs.no) & $1.15(0.77,1.71)$ & 0.499 & $0.90(0.57,1.41)$ & 0.633 \\
\hline $\begin{array}{l}\text { Length of index hospital stay } \\
\text { (every } 1 \text { day } \uparrow^{\mathrm{a}}\end{array}$ & $1.02(1.00,1.04)$ & 0.001 & $1.02(1.00,1.03)$ & 0.036 \\
\hline Hyponatremia (yes vs.no) & $2.21(1.29,3.79)$ & 0.004 & $1.85(1.06,3.24)$ & 0.031 \\
\hline Albumin (every $1 \mathrm{~g} / \mathrm{L} \uparrow$ ) & $0.96(0.93,0.99)$ & 0.017 & $0.97(0.94,1.01)$ & 0.104 \\
\hline
\end{tabular}

Variables that $p<0.1$ in univariate analysis and with clinical significance were included in multivariate analysis Abbreviations: OR odds ratio, $\mathrm{Cl}$ confidence interval, vs. verse, CVD cardiovascular disease

a Length of index hospital stay refers to the hospitalization days during which patients obtained peritoneal dialysis (PD) catheterization and began PD treatment 


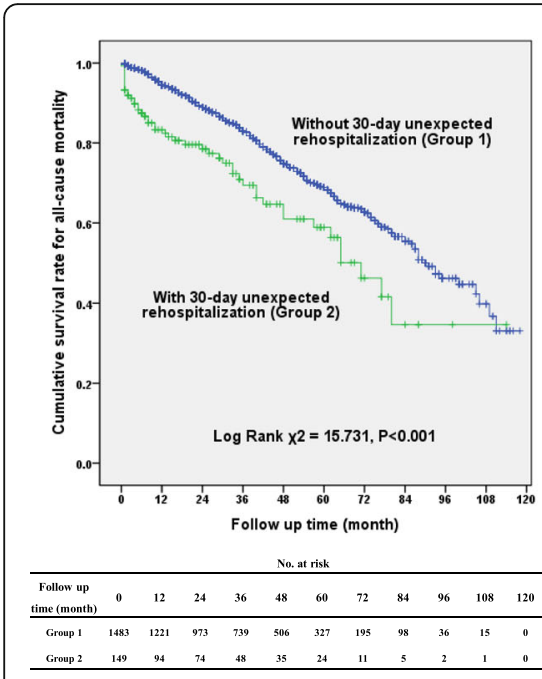

A: All-cause mortality

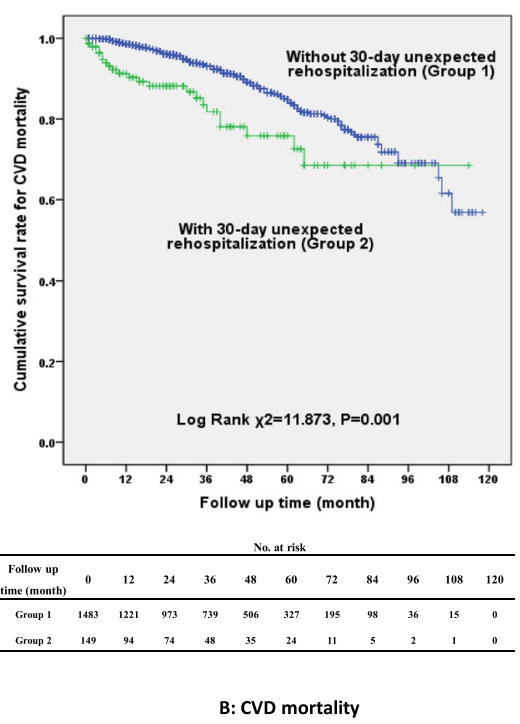

B: CVD mortality

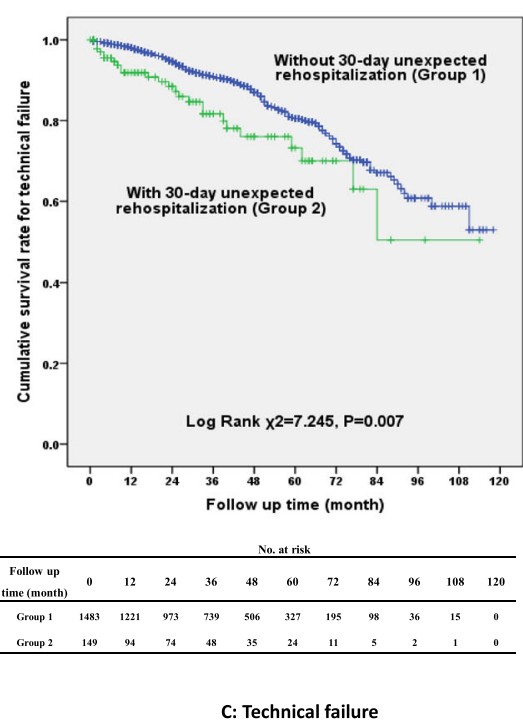

C: Technical failure

Fig. 3 Survival curves for patients without and with 30-day unexpected rehospitalization

of index hospital stay and hyponatremia were independently associated with 30-day unexpected rehospitalization. Furthermore, 30-day unexpected rehospitalization increased the risk of all-cause mortality and CVD mortality for incident PD patients.

Previous studies reported that rehospitalization rates were $15.8-37.5 \%$ in HD patients $[1,4,6,7,9]$ and $15.5-37.4 \%$ in PD patients $[1,4,5]$. Compared to previous studies, the

Table 3 Association between 30-day unexpected rehospitalization and outcomes in Cox regression models

\begin{tabular}{lll}
\hline Outcomes & HR $(\mathbf{9 5} \% \mathbf{C l})$ & $\boldsymbol{P}$ value \\
\hline $\begin{array}{ll}\text { All-cause mortality } \\
\text { Unadjusted }\end{array}$ & $1.83(1.35,2.49)$ & $<\mathbf{0 . 0 0 1}$ \\
Adjusted $^{\mathrm{a}}$ & $1.52(1.07,2.16)$ & $\mathbf{0 . 0 1 9}$ \\
CVD mortality & & \\
$\quad$ Unadjusted & $2.12(1.37,3.30)$ & $\mathbf{0 . 0 0 1}$ \\
Adjusted $^{b}$ & $1.73(1.03,2.90)$ & $\mathbf{0 . 0 3 8}$ \\
Technical failure $^{\text {Unadjusted }}$ & & \\
Adjusted $^{c}$ & $1.76(1.16,2.67)$ & $\mathbf{0 . 0 0 8}$ \\
\hline
\end{tabular}

Variables that $p<0.1$ in univariate analysis and with clinical significance were included in multivariate analysis

Abbreviations: $H R$ hazard ratio, $\mathrm{Cl}$ confidence interval, CVD cardiovascular disease, BMI body mass index, iPTH intact parathyroid hormone, TC total cholesterol, TG triglyceride

aVariables including age, gender, diabetes mellitus, CVD history, BMI, length of index hospital stay, hemoglobin, platelet, corrected calcium, phosphorus, iPTH, albumin, TC, TG, hyponatremia, hypokalemia, uric acid, creatinine were adjusted

bVariables including age, gender, diabetes mellitus, CVD history, BMI, length of index hospital stay, hemoglobin, platelet, corrected calcium, phosphorus, iPTH, albumin, TC, TG, uric acid, creatinine were adjusted

'Variables including age, gender, diabetes mellitus, CVD history, BMI, albumin, TG, hypokalemia were adjusted prevalence of 30-day unexpected rehospitalization in our study was much lower. One of the probable explanations might be that patients in our cohort were much younger than those in previous studies. In our study, the mean age was $46.9 \pm 15.3$ years old, while in the aforementioned studies, the mean age range was 57-66 years old. Older age was a risk factor for morbidity and mortality in the incident dialysis patients [21], and was also found to be an independent risk factor for unexpected rehospitalization for PD patients [5]. Secondly, the comorbid status of DM in our cohort was much less than that in the other studies. The proportion of diabetes in our patients was $25.6 \%$, while in Li Z's study, the proportion was $42.1 \%$ [5]. And in Ziv Harel's study, the proportion of patients with DM was as high as $62.0 \%$ [7]. $\mathrm{DM}$ is strongly associated with macro- and microvascular complications, including CVD, retinopathy, nephropathy, and neuropathy [22]. These complications might increase the risk of readmission. Thirdly, patients in our study were incident patients, while most of the previous studies included prevalent patients who had pretty long dialysis periods with more comorbidities and worse status $[1-4,6,7$, 9]. Moreover, the specific follow-up management strategies of our centre might also be attributable to the lower rate of unexpected rehospitalization [23-25]. In our center, patients were followed up 3 times within 1 month after discharged. The first follow-up was done by phone within 3 days to make sure that patients were familiar with PD operation and took medications as directed. The second follow-up was done by phone within 2 weeks to evaluate whether their dialysis prescriptions were appropriate, especially whether there was fluid overload. The third follow-up was done within 3-4 weeks by clinical visit to assess the comprehensive health condition after PD 
initiation. The patients could come back to visit their nephrologist through green channel, and a 24-h on-call service was always provided in the PD center to deal with their emergent problems. Additionally, as most of our patients were living in remote rural areas, we established a PD "satellite center" program across Guangdong Province to provide standardized training for doctors and nurses in satellite hospitals who provided cares to patients in remote areas [25]. The PD patients who had clinical symptoms in the early stage could be interventedtimely, which would prevent them from deteriorating to the point of admission [23]. All of the above factors may contribute to the decreased rate of the unexpected rehospitalization in our cohort.

We identified that length of index hospital stay was an independent risk factor of 30-day unexpected rehospitalization. Patients with longer hospital stay always presented with more severe or complicated disease condition during index admission [26]. Such complex condition not only prolonged their index hospital care, but also made them more vulnerable to an unexpected rehospitalization. Thus post-discharge care plan for these patients should be made carefully. Additionally, we revealed that hyponatremia was another risk factor of rehospitalization. It was reported that hyponatremia was associated with increased risk of 30-day rehospitalization among patients with congestive heart failure [27]. While in PD patients, hyponatraemia was always accompanied with hypokalaemia [28], which was a well-recognized risk parameter for peritonitis $[29,30]$. In addition, hyponatremia was reported to be a surrogate marker of longer hospital stay and poorer outcome for PD-related peritonitis [31]. Also, hyponatremia was reported to be related to a lower level of albumin [32], which would significantly increase the risk of peritonitis and other infectious diseases [33]. Moreover, hyponatremia always resulted from inappropriate water gain among PD patients [32, 34]. Persistent water retention would lead to severe fluid overload or refractory hypertension and finally cause rehospitalization. In addition, it was demonstrated that hyponatremia was strongly correlated with the decline of residual renal function (RRF) [35], which is a well-recognized risk factor of fluid overload [17]. In particular, hyponatremia has been found to be significantly associated with an increased risk of infectionrelated hospitalization and new-onset CVD events for dialysis patients [36, 37]. All of the above factors increased the risk of rehospitalization for incident PD patients.

It has been reported that rehospitalizations were associated with increased morbidity and mortality and reduced quality of life among dialysis population [1-3]. In the current study, we revealed that 30-day unexpected rehospitalization was independently associated with poor long-term outcomes of incident PD patients. A probable explanation for this finding is that the adverse clinical events that cause early unexpected rehospitalization might also lead to worse prognosis. First, peritonitis was the most common cause of rehospitalization in our study. It is well known that peritonitis could lead to the failure of peritoneal function, resulting in transferring to HD or even death $[16,38]$. Our previous researches demonstrated that early onset peritonitis in incident PD patients affected not only the peritoneal function but also the confidence and compliance of the patients in the treatment modality, which in turn led to worse outcomes [33]. Second, catheter malfunction was the other important cause of rehospitalization, accounting for $1.8 \%$ of our study population, which also was an important cause of early technical failure of PD. Although the prevalence of catheter malfunction was relatively low in our centre, it was still an important cause of 30-day unexpected rehospitalization. Peritonitis and catheter failure were the most common causes of readmission which related to patient education and experience of surgeon. Previous studies by our colleagues have shown that lower education level is associated with the first episode of peritonitis [29] and long-term all-cause mortality [39]. We also found that severe fluid overload was another important reason for rehospitalization in this cohort, accounting for $1.2 \%$ of our study population. Fluid overload not only played an inverse role in the preservation of RRF [17, 40] but also increased both all-cause and CVD mortality [17]. All of these aforementioned events could lead to poor outcomes. The rest of the rehospitalization reasons, such as CVD events, non-peritonitis infection, etc., would also result in a poor prognosis undoubtedly $[3,20]$.

Our study has several limitations. First, all of the data were collected from a single centre. The results may not be generalizable to other centres. Second, we were not able to consider all variables potentially associated with the rehospitalization and long-term outcomes. Further studies that take into account more risk factors, such as post-discharge medical care, health literacy and social support, are warranted. Nonetheless, to our knowledge, this was the first study concerning 30-day unexpected rehospitalization in a large cohort of incident PD patients in developing country. The strengths of our study included its large cohort and complete follow-up data. The results of our study might be of value in guiding clinical practice.

\section{Conclusions}

In summary, the prevalence of 30-day unexpected rehospitalization for incident PD patients in our centre was 9.1\%. The top three causes for the rehospitalization were PDrelated peritonitis, catheter malfunction and severe fluid 
overload. Length of index hospital stay and hyponatremia were independently associated with the rehospitalization. Thirty-day unexpected rehospitalization increased the risk of all-cause mortality and CVD mortality for PD patients.

\section{Abbreviations}

ESRD: End stage renal disease; PD: Peritoneal dialysis; CVD: Cardiovascular disease; OR: Odds ratio; Cl: Confidence interval; HR: Hazard ratio; USRDS: United States Renal Data System; HD: Hemodialysis; DM: Diabetes mellitus; BMI: Body mass index; iPTH: Intact parathyroid hormone; TC: Total cholesterol; TG: Triglyceride; SD: Standard deviation; IQR: Interquartile range; RRF: Residual renal function

\section{Acknowledgements}

We are grateful to all nephrologists and nurses in our PD center for their helpful assistance.

\section{Authors' contributions}

$J B L$ and JY contributed equally to the study, they contributed to the study design, data collection and analyses, and wrote the manuscript. NYH, HJY, $D W, Y P, X B G, C Y Y$ contributed to the data collection and analyses. XY contributed to study design, data analysis and revision of the manuscript. XQY conceived and designed the study, wrote the manuscript and provided final supervision. All authors read and approved the final version of the manuscript.

\section{Funding}

This work was supported in part by the National Key Research and Development Project (Grant no. 2016YFC0906101); the Operational Grant of Guangdong Provincial Key Laboratory (Grant no. 2014B030301023, 2017B030314019); the Key Laboratory of National Health Commission and Key Laboratory of Nephrology, Guangdong Province, Guangzhou, China (Grant no. 2002B60118); the Guangdong Provincial Programme of Science and Technology (Grant no. 2017A050503003, 2017B020227006); the Guangzhou Municipal Programme of Science and Technology (Grant no. 201704020167); the Natural Science Foundation of China (Grant no. 81774069,81600545) and the Natural Science Foundation of Guangdong Province, China (Grant no.2017A030310199).

\section{Availability of data and materials}

The data used in the current study are available from the corresponding author on reasonable request.

\section{Ethics approval and consent to participate}

The research was conducted ethically in accordance with the World Medical Association Declaration of Helsinki and was approved by the Ethics Committee of The First Affiliated Hospital, Sun Yat-sen University. All participants were asked for a permission of using their medical data for a noncommercial study, and written informed consent was obtained from them at the initiation of PD follow-up.

\section{Consent for publication}

Not applicable.

\section{Competing interests}

The authors declare that they have no competing interests.

\section{Author details}

'Department of Nephrology, The First Affiliated Hospital, Sun Yat-sen University, Guangzhou 510080, Guangdong, China. ${ }^{2}$ Key Laboratory of Nephrology, National Health Commission and Guangdong Province, Guangzhou 510080, Guangdong, China. 'Department of Statistical Science, School of Mathematics, Sun Yat-sen University, Guangzhou 510275, Guangdong, China. ${ }^{4}$ Guangdong Provincial People's Hospital, Guangzhou 510080, Guangdong, China. ${ }^{5}$ School of Medicine, South China University of Technology, Guangzhou 510080, Guangdong, China.
Received: 2 May 2020 Accepted: 3 December 2020

Published online: 06 January 2021

\section{References}

1. Saran R, Robinson B, Abbott KC, Agodoa LYC, Bragg-Gresham J, Balkrishnan R, et al. US renal data system 2018 annual data report: epidemiology of kidney disease in the United States. Am J Kidney Dis. 2019;73(3S1):A7-8.

2. Plantinga LC, King L, Patzer RE, Lea JP, Burkart JM, Hockenberry JM, et al. Early hospital readmission among hemodialysis patients in the United States is associated with subsequent mortality. Kidney Int. 2017:92(4):934-41.

3. Laurin LP, Harrak H, Elftouh N, Ouimet D, Vallée M, Lafrance JP. Outcomes of infection-related hospitalization according to dialysis modality. Clin J Am Soc Nephrol. 2015;10(5):817-24.

4. Perl J, MCArthur E, Bell C, Garg AX, Bargman JM, Chan CT, et al. Dialysis modality and readmission following hospital discharge: a population-based cohort study. Am J Kidney Dis. 2017;70(1):11-20.

5. Li Z, Abreu Z, Penner T, He L, Liu X, Bargman JM. Analysis of hospitalization after peritoneal dialysis catheter implantation. Perit Dial Int. 2016;36(5):540-6.

6. Flythe JE, Katsanos SL, Hu Y, Kshirsagar AV, Falk RJ, Moore CR. Predictors of 30-day hospital readmission among maintenance hemodialysis patients: a hospital's perspective. Clin J Am Soc Nephrol. 2016;11(6):1005-14.

7. Harel Z, Wald R, McArthur E, Chertow GM, Harel S, Gruneir A, et al. Rehospitalizations and emergency department visits after hospital discharge in patients receiving maintenance hemodialysis. J Am Soc Nephrol. 2015;26(12):3141-50.

8. Erickson KF, Winkelmayer WC, Chertow GM, Bhattacharya J. Physician visits and 30-day hospital readmissions in patients receiving hemodialysis. J Am Soc Nephrol. 2014;25(9):2079-87.

9. Chan L, Chauhan K, Poojary P, Saha A, Hammer E, Vassalotti JA, et al. National estimates of 30-day unplanned readmissions of patients on maintenance hemodialysis. Clin J Am Soc Nephrol. 2017;12(10):1652-62.

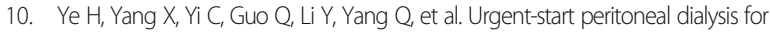
patients with end stage renal disease: a 10-year retrospective study. BMC Nephrol. 2019;20:238.

11. Ouyang CJ, Huang FX, Yang QQ, Jiang ZP, Chen W, Qiu Y, et al. Comparing the incidence of catheter-related complications with straight and coiled Tenckhoff catheters in peritoneal dialysis patients-a single-center prospective randomized trial. Perit Dial Int. 2015;35:443-9.

12. Go AS, Chertow GM, Fan D, McCulloch CE, Hsu CY. Chronic kidney disease and the risks of death, cardiovascular events, and hospitalization. N Engl J Med. 2004;351(13):1296-305.

13. Pan WC, Lau W, Mattman A, Kiaii M, Jung B. Comparison of hypoalbuminemia-corrected serum calcium using BCP albumin assay to ionized calcium and impact on prescribing in hemodialysis patients. Clin Nephrol. 2018;89(1):34-40.

14. Shavit L, Merin O, Grenader T, Jacobson E, Waldenberg C, Bitran D, et al. Hyponatremia predicts poor outcomes in patients with chronic kidney disease undergoing heart operation. Ann Thorac Surg. 2018;106(3):696-701.

15. Nakhoul GN, Huang H, Arrigain S, Jolly SE, Schold JD, Nally JV, et al. Serum potassium, end-stage renal disease and mortality in chronic kidney disease. Am J Nephrol. 2015;41(6):456-63.

16. Li PK, Szeto CC, Piraino B, de Arteaga J, Fan S, Figueiredo AE, et al. ISPD peritonitis recommendations: 2016 update on prevention and treatment. Perit Dial In. 2016;36(5):481-508.

17. Guo Q, Lin J, Li J, Yi C, Mao H, Yang X, et al. The effect of fluid overload on clinical outcome in southern Chinese patients undergoing continuous ambulatory peritoneal dialysis. Perit Dial Int. 2015;35(7):691-702.

18. Livingston EH. What is an abdominal wall hernia? JAMA. 2016;316(15):1610.

19. Siddiqui M, Calhoun DA. Refractory versus resistant hypertension. Curr Opin Nephrol Hypertens. 2017;26(1):14-9.

20. Wu X, Yang X, Liu X, Yi C, Guo Q, Feng X, et al. Patient survival and technique failure in continuous ambulatory peritoneal dialysis patients with prior stroke. Perit Dial Int. 2017;26(1):14-9.

21. Brown EA, Finkelstein FO, lyasere OU, Kliger AS, et al. Peritoneal or hemodialysis for the frail elderly patient, the choice of 2 evils? Kidney Int. 2017;91(2):294-303.

22. Forbes JM, Fotheringham AK. Vascular complications in diabetes: old messages, new thoughts. Diabetologia. 2017;60(11):2129-38.

23. Yu X, Yang X. Peritoneal dialysis in China. Meeting the challenge of chronic kidney failure. Am J Kidney Dis. 2015;65(1):147-51.

24. Yu X, Yang X, Huang N. Management of a rapidly growing peritoneal dialysis population at the First Affiliated Hospital of Sun Yat-sen University. Perit Dial Int. 2014;34(Suppl 2):S31-4. 
25. Jiang Z, Yu X. Advancing the use and quality of peritoneal dialysis by developing a peritoneal dialysis satellite center program. Perit Dial Int. 2011;31:121-6.

26. Springel T, Laskin B, Furth S. Readmission within 30 days of hospital discharge among children receiving chronic dialysis. Clin J Am Soc Nephrol. 2014;9:536-42.

27. Donzé JD, Beeler PE, Bates DW. Impact of hyponatremia correction on the risk for 30-day readmission and death in patients with congestive heart failure. Am J Med. 2016;129(8):836-42.

28. Zanger R. Hyponatremia and hypokalemia in patients on peritoneal dialysis. Semin Dial. 2010;23(6):575-80.

29. Fan $X$, Huang $R$, Wang J, Ye H, Guo Q, et al. Risk factors for the first episode of peritonitis in Southern Chinese continuous ambulatory peritoneal dialysis patients. PLoS One. 2014;9(9):e107485

30. Shu KH, Chang CS, Chuang YW, Chen CH, Cheng CH, Wu MJ, et al. Intestinal bacterial over growth in CAPD patients with hypokalaemia. Nephrol Dial Transplant. 2009;24(4):1289-92.

31. Tseng MH, Cheng CJ, Sung CC, Chou YC, Chu P, et al. Hyponatremia is a surrogate marker of poor outcome in peritoneal dialysis-related peritonitis. BMC Nephrol. 2014;15:113.

32. Kang SH, Cho KH, Park JW, Yoon KW, Do JY. Characteristics and clinical outcomes of hyponatraemia in peritoneal dialysis patients. Nephrology (Carlton). 2013;18(2):132-7.

33. Wu H, Huang R, Yi C, Wu J, Guo Q, Zhou Q, et al. Risk factors for early-onset peritonitis in southem Chinese peritoneal dialysis patients. Perit Dial Int. 2016;36(6):640-6.

34. Zevallos G, Oreopoulos DG, Halperin ML. Hyponatremia in patients undergoing CAPD: role of water gain and/or malnutrition. Perit Dial Int. 2001;21(1):72-6

35. Dimitriadis C, Sekercioglu N, Pipili C, Oreopoulos D, Bargman JM. Hyponatremia in peritoneal dialysis: epidemiology in a single centre and correlation with clinical and biochemical parameters. Perit Dial Int. 2014;34(3):260-70.

36. Mandai S, Kuwahara M, Kasagi Y, Kusaka K, Tanaka T, Shikuma S, et al. Lower serum sodium level predicts higher risk of infection-related hospitalization in maintenance hemodialysis patients: an observational cohort study. BMC Nephrol. 2013;14:276.

37. Kim HW, Ryu GW, Park CH, Kang EW, Park JT, Han SH, et al. Hyponatremia predicts new-onset cardiovascular events in peritoneal dialysis patients. PLoS One. 2015;10(6):e0129480.

38. Ye H, Zhou Q, Fan L, Guo Q, Mao H, Huang F, et al. The impact of peritoneal dialysis-related peritonitis on mortality in peritoneal dialysis patients. BMC Nephrol. 2017;18(1):186.

39. Xia X, Qiu Y, Yu J, Lin T, Lu M, Yi C, Lin J, Ye H, Chen W, Mao H, Yang X, Huang F. Ten-year survival of patients treated with peritoneal dialysis: a prospective observational cohort study. Perit Dial Int. 2020;40:573-80.

40. Tian N, Guo Q, Zhou Q, Cao P, Hong L, Chen M, et al. The impact of fluid overload and variation on residual renal function in peritoneal dialysis patient. PLoS One. 2016;11(4):e0153115.

\section{Publisher's Note}

Springer Nature remains neutral with regard to jurisdictional claims in published maps and institutional affiliations.

Ready to submit your research? Choose BMC and benefit from:

- fast, convenient online submission

- thorough peer review by experienced researchers in your field

- rapid publication on acceptance

- support for research data, including large and complex data types

- gold Open Access which fosters wider collaboration and increased citations

- maximum visibility for your research: over $100 \mathrm{M}$ website views per year

At $\mathrm{BMC}$, research is always in progress.

Learn more biomedcentral.com/submissions 